\title{
Heat exchangers of increased thermal efficiency for power and technological machines: development and research
}

\author{
Nikolai Chernov ${ }^{1, *}$ \\ ${ }^{1}$ Togliatti State University, Institute of Mechanical Engineering, 445020, Belorusskaya str., 14, \\ Togliatti, Russia
}

\begin{abstract}
The paper reviews the development and application of new technologies and designs of heat exchangers, as well as the ways to increase the efficiency of heat exchange, with the aim to improve the thermal engineering parameters and performance characteristics of power and process machines and to reduce toxic emissions during their manufacture. Theoretical and practical foundations for increasing the heat exchange efficiency of heat exchangers are also reviewed. The increase in heat exchange efficiency of heat exchangers is achieved by increasing the area of heat exchange surfaces in contact with heat carriers by forming a macrorelief having optimal geometric parameters by finning. A highefficiency method of machining, deforming cutting (DC), which is based on cutting and bending of the layers of the surface layer of the workpiece metal, providing a wide range of the resulting macrorelief, is chosen for finning the heat exchange surfaces. The paper provides optimal geometric parameters of finning: the fin height $(\mathrm{H})$, the fin pitch $(\mathrm{S})$ and the thickness of the rib (a). The efficiency of the selected geometric parameters is researched and experimentally tested. It has been experimentally established that the finest finning parameters are the minimum fin pitch and the maximum possible fin height. The interrelation is established, and the geometrical parameters of the heat exchange surface processed by the DC method are determined with the operational characteristics of heat exchangers. The greatest thermal efficiency was achieved with a fin pitch of $\mathrm{S}=1.5 \mathrm{~mm}$, a fin height of $\mathrm{h}=3 \mathrm{~mm}$, and a thickness of $\mathrm{a}=0.75 \mathrm{~mm}$. We consider the TA (water - oil) design developed by the author to enhance the efficiency of heat exchange. The research results on increasing the efficiency of heat transfer are presented in the paper. The application of the finned coiled tubing heat exchanger developed on the basis of the research results makes it possible to significantly improve the efficiency, reliability, and service life of power and process machines.
\end{abstract}

* Corresponding author: ns-chernov@ mail.ru 


\section{Introduction}

In modern hydraulic drives, power and technological machines (test benches, hydraulic presses, machine tools, automatic lines for metalworking), the most common are recuperative heat exchangers (HE), in which one side of the heat exchange surface is washed with a hot medium - working fluid (WF), and the other one - with a coolant. From one medium to another, heat is transferred through the wall separating them from the heatconducting material.

In the technological machines for the production of VAZ vehicles, up to now, tubular HE (smooth tubes) from Vickers (Germany) of the OCW series have been used, as well as the tubular HE of own manufacture.

The tubular HE (depending on the size) contains 24 or 32 smooth brass tubes of $10 \mathrm{~mm}$ or $12 \mathrm{~mm}$ in diameter soldered to the tube boards, i.e., includes 48 or 64 brazed joints.

Expansion and updating of the model range of the VAZ cars and the formation of the production of their own technological and power machines for their manufacture, required creating new technologies and, based on them, highly efficient HE of various designs in place of imported ones. HE designs in each specific case should provide an optimal combination of thermal efficiency, small dimensions, and weights, increase the efficiency of heat exchange, operational reliability, and service life, and ensure reduction of toxic emissions into the atmosphere during their manufacture. The creation of perfect HE designs determines the search for highly efficient heat exchange surfaces by using finned tubes with a sufficiently large heat exchange surface as compared to smooth cylindrical tubes.

An increase in the intensity of $\mathrm{HE}$, heat exchange can be achieved by increasing the area of heat exchange surfaces in contact with heat carriers by forming a macrorelief having optimal geometric parameters.

Increasing the efficiency of heat exchange is one of the main ways to improve the thermal characteristics of HE cooling systems for power and process vehicles.

The most effective and economically advantageous for power machines are heat exchangers with coiled radiators from finned tubes. When designing these heat exchangers, developed calculation methods are used to select the best fin parameters, characterized by the height (h) and hickness (a) of the fins, as well as the fin pitch (S) [1].

Intensification of heat transfer processes leads to an increase in the heat transfer coefficient, which for clean heat exchange surfaces depends on the heat transfer coefficients on the side of the heating and heating media.

Taking into account operational and technical capabilities, the heat transfer intensification should be carried out on the side of the heat carrier, which has a small value of the heat transfer coefficient on both sides of the heat exchange surface. It is associated with an increase in energy costs to overcome the increasing hydraulic resistance and is due to energy efficiency [4].

At present, there are the following basic methods for intensifying heat transfer:

- Constructing rough surfaces and surfaces of complex shape that promote turbulence in the wall layer;

- Using turbulent inserts in channels;

- Increasing the surface area of heat transfer by finning;

- Influencing the heat carrier flow by electric, magnetic, and ultrasonic fields;

- Mechanical effects on the heat transfer surface by rotating and vibrating it;

- Adding solids or gas bubbles to the coolant.

The possibility and expediency of applying this or that method of intensification for specific conditions are determined by technical capabilities and their effectiveness. The most widely used in machines is the intensification of heat exchange, achieved by finning 
the outer surface of coils, with the condition that a coolant with a low value of the heat transfer coefficient is directed into the intertubular space.

The combination of the ability to select the geometry of the surface layer at the macro level and the use of modern cutting technology allows to create surface structures with controlled properties of operational reliability, antifriction, and increased efficiency of heat transfer. High efficiency and a wide range of standard sizes of the obtained macrorelief is provided by a method based on cutting and bending the layers of the surface layer of the workpiece metal. It has the title of deforming cutting (DC) and is based on the use of conventional metal-cutting equipment, a simple tool, as well as it is non-waste (1).

\section{Theoretical bases of designing}

\subsection{Choosing the geometric parameters of the surface fins of HE elements}

The surface finning parameters are shown in Fig. 1.

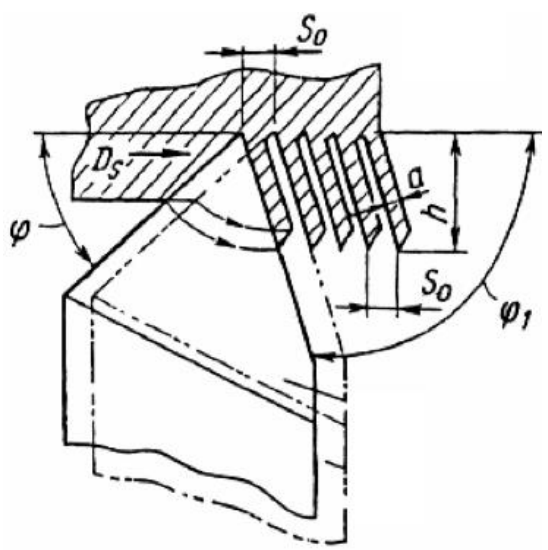

Fig. 1 Geometrical parameters of the finned surface of the projected heat exchangers.

The choice of geometric parameters of the fin is carried out by selecting the appropriate tools and technological processing modes. Thus, the change in the fin pitch $(\mathrm{S})$ is achieved by the value of the tool feed per one revolution of the workpiece $S$; control over the thickness of the edge (a) is achieved by selecting the main tool angle $\varphi$; the fin height (h) is varied by the cutting depth $t$ at the selected main cutting angle of the tool $(\varphi)$.

In practice, when processing fins, due to the presence of their deformations, they become thicker or thinner than the theoretical thickness. This change is accounted for by the edge distortion coefficient $(\xi)$. Therefore, to produce edges of a given thickness (a), the tool is made with an angle $\varphi$, being calculated according to formula:

$$
\varphi=\arcsin \left(\frac{a}{S \cdot \xi}\right)
$$

where: $\xi$ - the coefficient depending on the properties of the material being processed, the machining modes, the geometry of the tool, its position relative to the axis of rotation of the part, and the pipe diameter.

According to the research results, this coefficient for copper, copper alloys, and steel is $0.88-1.15$. For aluminum, the coefficient $\xi$ can increase to 1.23 . The possibility of using the minimum values of the angle $\varphi$ depends on the ductility and strength of the material being processed. 
According to the results of experimental studies, the minimum permissible values of $\varphi$ can be as follows: for aluminum $-15^{\circ}$; for copper and copper alloys $-21^{\circ}$; for corrosionresistant steels $-33^{\circ}$ and for medium-carbon steels $-39^{\circ}$.

In some cases, it is required to provide an undefined fin thickness (a), with the fin pitch being selected (S), as well as the specified groove width (b). In this case, the choice of the angle $\varphi$ is made by the formula:

$$
\varphi=\arcsin \left[\frac{1}{\xi}\left(\sin \varphi_{1}-\frac{b}{S}\right)\right]
$$

The slope of the edges to the base depends on the auxiliary angle of the tool $\varphi_{1}$. To obtain a given slope of the edge, the auxiliary angle of the tool $\varphi_{1}$ is chosen to be $90^{\circ} \pm \psi$. A deviation of $\varphi_{1}$ from $90^{\circ}$ in one direction or another results in a decrease in the fin height (h) and the width of the gap (b) between the fins. For a number of cases, the verticality of the edges $(\psi=0)$ is required, when $\varphi_{1}$ is $90^{\circ}$. In this case, the reduction in pitch (S) proportionately decreases the width of the groove (b).

The specified fin height $(\mathrm{h})$ is provided by choosing the cutting depth $\mathrm{t}$ by the formula:

$$
t=\left[h \xi-\frac{S \cos (\varphi-\psi)}{2}\right] \cdot\left(\frac{\sin \varphi}{\cos \psi}\right)
$$

For vertical edges, the formula is simplified:

$$
t=(h \xi-0,5 S \cos \varphi) \cdot(\sin \varphi)
$$

When working out the technology of manufacturing elements of heat exchangers using the method of deforming cutting, it was experimentally established that the fin thickness (a) should be half the pitch (S). For copper, the fin height is in the range of $2 \ldots 3 \mathrm{~mm}$, and the fin pitch is $1.5 \ldots 2.5 \mathrm{~mm}$.

Due to the fact that the finning parameters primarily affect the value of the heat transfer surface, it can be noted before the calculations that the best values are the maximum values: the maximum rib height and the minimum fin pitch. But increasing the height of the ribs leads to a decrease in the heat transfer coefficient, so it is necessary to perform verification calculations.

\section{Practical basics of design}

\subsection{Methods of research}

For research, the author developed a new design of the water-oil coil heat exchanger (Figure 2.1).

The method of carrying out experimental studies is to compare and analyze the evaluation of the characteristics of HE radiators with smooth pipes and coils with finned.

As a basic design, the heat exchanger for carrying out comparative tests is used with a radiator made of smooth pipes, which is used in the technological equipment of VAZ. The heat exchanger is a radiator with 44 parallel brass smooth tubes with a diameter of $10 \mathrm{~mm}$, a wall thickness of $1 \mathrm{~mm}$, and a length of $350 \mathrm{~mm}$. The arrangement of the tubes in the heat exchanger is a checkerboard with a pitch between the rows of tubes of $12 \mathrm{~mm}$. The coolantcoolant flows in parallel inside all 44 tubes, the oil flushes the tubes from the outside. The surface area of the heat exchanger for the basic design of the radiator is $0.47 \mathrm{~m}^{2}$. The area of the pass-through section of the cooling liquid is $22.1 \mathrm{~cm}^{2}$. 


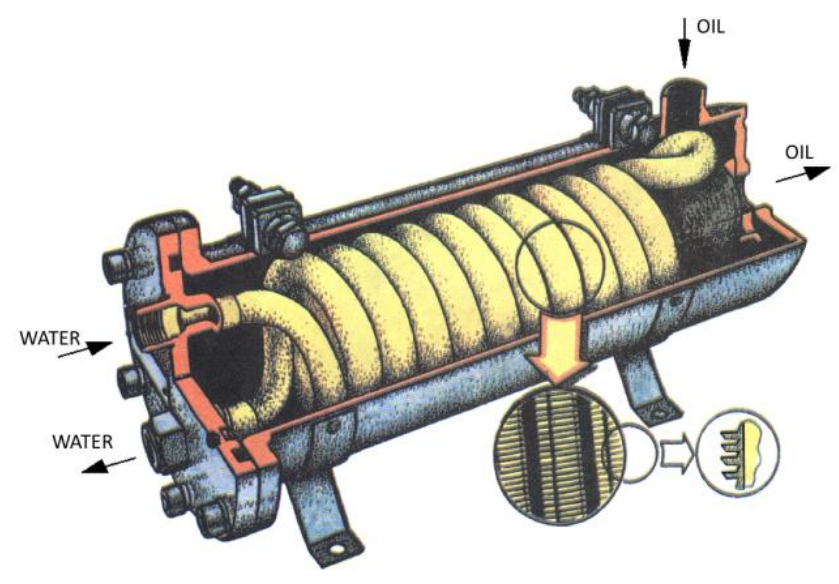

Fig. 2. A heat exchanger with a finned tube coil.

Common in the design of the basic and new heat exchanger is a casing with an internal diameter of $110 \mathrm{~mm}$. To assess the effectiveness of HE, it is necessary to ensure a constant flow of oil and liquid during the testing of basic and new designs. The flow rate of the cooling liquid is not less than $40 \%$ of the flow rate of the cooled liquid (oil).

The thermophysical parameters of the temperature drop of the cooled and cooling liquid at the inlet and outlet of the heat exchangers were obtained at the test stand and the heat exchanger.

To assess the efficiency of the heat exchangers being compared:

$t_{B x}^{B}$ - coolant temperature at the inlet;

$t_{\text {Bblx }}^{B}$ - coolant temperature at the outlet;

$t_{s x}^{\mathcal{M}}$ - inlet oil temperature;

$t_{\text {sblx }}^{M}$ - outlet oil temperature;

$\mathrm{G}_{\mathrm{M}}$ - mass flow of oil, $\mathrm{kg} / \mathrm{s}$;

$\mathrm{G}_{\mathrm{B}}$ - mass flow of coolant, $\mathrm{kg} / \mathrm{s}$.

Calculations were carried out based on the results of three or four measurements of the indicated values with an interval of 10-15 minutes:

The coefficient of the heat exchanger efficiency (the ratio of the actual transferred specific heat $Q_{M}$ to its limiting quantity $Q_{n p e d}$.

$$
\eta_{э \phi}=\frac{Q_{м}}{Q_{\text {пред }}}
$$

The calculation of $\eta_{\ni}$ was performed for the basic and new designs, and the closer the value of $\eta_{\ni \phi}$ to unity, the more efficient heat exchange is provided by this heat exchanger structure.

\section{Determining the HE performance characteristics when changing the geometric parameters of finning}

4. 1. To assess the influence of the fin pitch on the HE characteristics during the test, the following parameters are used: 
$\boldsymbol{t}_{\mathbf{1}}^{\prime}$ - the temperature of the oil to be cooled at the HE inlet, ${ }^{\circ} \mathrm{C}$;

$\boldsymbol{t}_{2}^{\prime}$ - temperature of heated water at the inlet to $\mathrm{HE},{ }^{\circ} \mathrm{C}$;

$\mathrm{V}_{1}$ - volumetric flow of oil, $1 / \mathrm{min}$;

$\mathrm{V}_{2}$ - volume flow of water, $1 / \mathrm{min}$;

$\mathrm{h}$ - the fin height, $\mathrm{mm}$;

$\mathrm{S}$ - fin pitch, $\mathrm{mm}$.

$\boldsymbol{t}_{1}^{\prime}=55^{\circ} \mathrm{C}$ and $\boldsymbol{t}_{1}^{\prime}=90^{\circ} \mathrm{C} ; \boldsymbol{t}_{2}^{\prime}=12^{\circ} \mathrm{C} ; \mathrm{V}_{1}=1401 / \mathrm{min} ; \mathrm{V}_{2}=401 / \mathrm{min} ; \mathrm{h}=2 \ldots 3 \mathrm{~mm} ; \mathrm{S}=$ $1,5 \ldots 2,5 \mathrm{~mm}$

Table 1. The values of the HE parameters from the fin pitch at $t_{1}^{\prime}=55^{\circ} \mathrm{C}$

\begin{tabular}{|c|c|c|c|c|}
\hline $\mathbf{S}, \mathbf{m m}$ & $\mathbf{Q}, \mathbf{k W}$ & $\mathbf{K}, \mathbf{W} /\left(\mathbf{m}^{\mathbf{2}} \cdot \mathbf{K}\right)$ & $\boldsymbol{\eta}^{\text {э中 }}$ & $\mathbf{F}, \mathbf{m}^{\mathbf{2}} / \mathbf{m}$ \\
\hline 1,5 & 28,22 & 612,5 & 0,344 & 0,399 \\
\hline 1,75 & 26,19 & 634,3 & 0,325 & 0,349 \\
\hline 2,00 & 24,51 & 651,6 & 0,311 & 0,312 \\
\hline 2,25 & 23,10 & 665,8 & 0,298 & 0,283 \\
\hline 2,50 & 21,88 & 677,5 & 0,587 & 0,260 \\
\hline
\end{tabular}

Table 2. The values of the parameters HE from the pitch of the fin at $t_{1}^{\prime}=90^{\circ} \mathrm{C}$

\begin{tabular}{|c|c|c|c|c|}
\hline $\mathbf{S}, \mathbf{m m}$ & $\mathbf{Q}, \mathbf{k W}$ & $\mathbf{K}, \mathbf{W} /\left(\mathbf{m}^{\mathbf{2}} \cdot \mathbf{K}\right)$ & $\boldsymbol{\eta}^{\text {э中 }}$ & $\mathbf{F}, \mathbf{~ m}^{\mathbf{2}} / \mathbf{m}$ \\
\hline 1,5 & 70,22 & 839,0 & 0,456 & 0,399 \\
\hline 1,75 & 65,77 & 873,2 & 0,396 & 0,349 \\
\hline 2,00 & 63,00 & 904,6 & 0,378 & 0,312 \\
\hline 2,25 & 58,78 & 929,0 & 0,364 & 0,283 \\
\hline 2,50 & 55,96 & 949,3 & 0,351 & 0,260 \\
\hline
\end{tabular}

where:

$\mathbf{Q}$ - thermal power;

$\mathbf{K}$ - heat transfer coefficient;

$\boldsymbol{\eta}^{\text {э }}$ - thermal efficiency coefficient;

$\mathbf{F}$ - heat transfer surface area, $\mathrm{m} 2 / \mathrm{m}$, referred to one meter of coil length.

The Figures 3.1, 3.2, 3.3 show the dependence of the heat output and the heat transfer coefficient on the variation of the fin pitch.

The Figures 3.1, 3.2, 3.3 show the dependence of the heat output and the heat transfer coefficient on the variation of the fin pitch. 


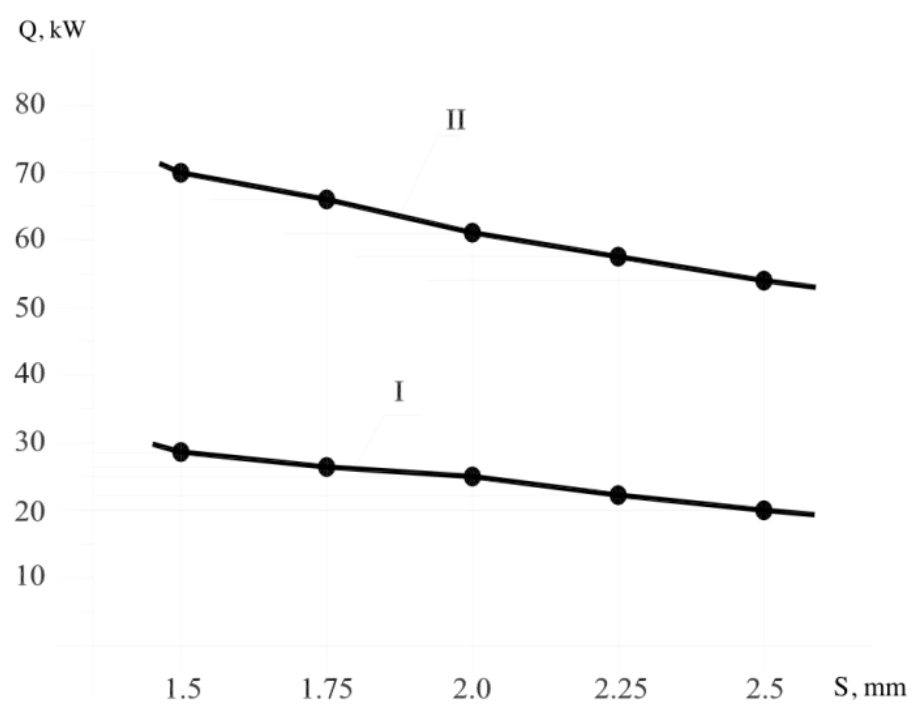

Fig. 3.1 The dependence of the heat output of the heat exchanger on the variation of the fin pitch: I with $\dot{t}_{1}^{\prime}=55^{\circ} \mathrm{C}$, II - with $\dot{t}_{1}^{\prime}=90^{\circ} \mathrm{C}$.

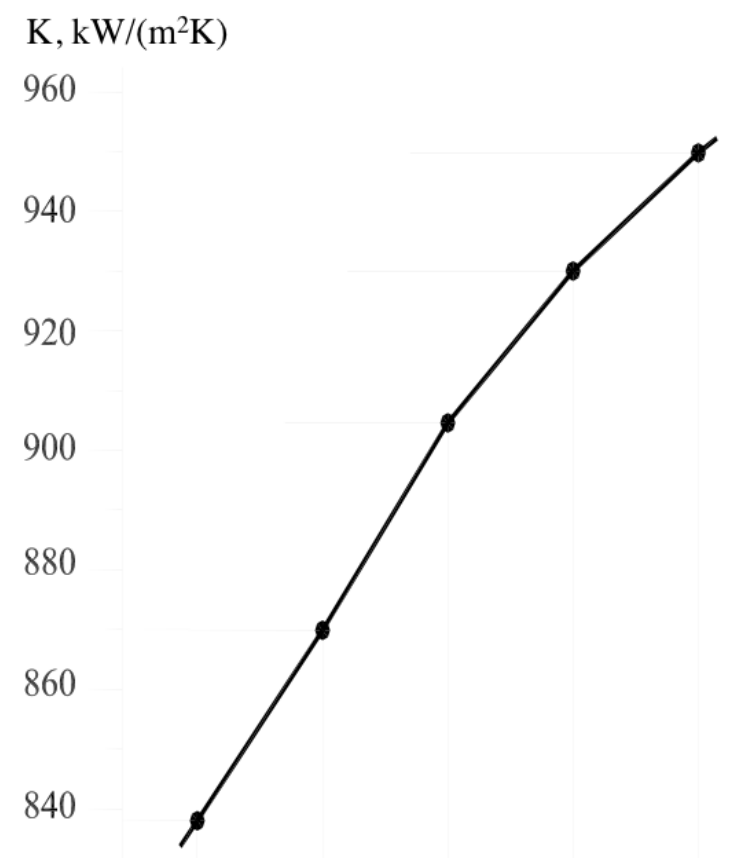

\section{$\begin{array}{llllll}1.5 & 1.75 & 2.0 & 2.25 & 2.5 \mathrm{~S}, \mathrm{~mm}\end{array}$}

Fig. 3.2 The dependence of the heat transfer coefficient on the variation of the fin pitch, with $t_{1}^{\prime}=55^{\circ}$ C. 


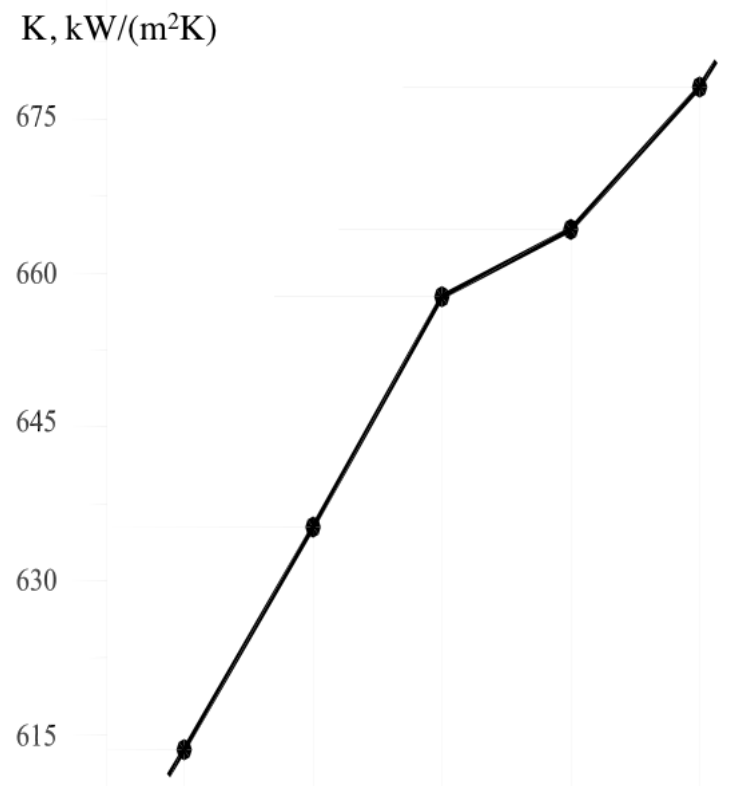

$$
\begin{array}{llllll}
1.5 & 1.75 & 2.0 & 2.25 & 2.5 & \mathrm{~S}, \mathrm{~mm}
\end{array}
$$

Fig. 3. 3 The dependence of the heat transfer coefficient on the variation of the fin pitch, with $t_{\mathbf{1}}^{\prime}=$ $90^{\circ} \mathrm{C}$.

\section{2 To estimate the height of the ribs on the characteristics of HE, the parameters:}

$t_{1}^{\prime}=55^{\circ} \mathrm{C}$ and $t_{1}^{\prime}=90^{\circ} \mathrm{C} ; t_{2}^{\prime}=12^{\circ} \mathrm{C} ; \mathrm{V}_{1}=140 \mathrm{l} / \mathrm{min} ; \mathrm{V}_{2}=40 \mathrm{l} / \mathrm{min} ;$

$\mathrm{h}=2 \ldots 3 \mathrm{~mm} ; \mathrm{S}=1,5 \mathrm{~mm}$.

The results of the calculations are summarized in Table. 3.3 and 3.4

Table 3. The values of $\mathrm{HE}$ parameters from the rib height at $t_{1}^{\prime}=55^{\circ} \mathrm{C}$

\begin{tabular}{|c|c|c|c|c|}
\hline $\mathbf{h}, \mathbf{m m}$ & $\mathbf{Q}, \mathbf{k W}$ & $\mathbf{K}, \mathbf{W} /\left(\mathbf{M}^{\mathbf{2}} \mathbf{K}\right)$ & $\mathbf{\eta}^{\text {э中 }}$ & $\mathbf{F}, \mathbf{m}^{\mathbf{2}} / \mathbf{m}$ \\
\hline 2,00 & 27,88 & 646,5 & 0,345 & 0,372 \\
\hline 2,25 & 28,30 & 604,6 & 0,345 & 0,405 \\
\hline 2,50 & 29,70 & 568,5 & 0,348 & 0,438 \\
\hline 2,75 & 30,70 & 537,1 & 0,350 & 0,469 \\
\hline 3,00 & 31,50 & 510,2 & 0,352 & 0,500 \\
\hline
\end{tabular}

Table 4. The values of the $\mathrm{HE}$ parameters from the height of the edges at $t_{1}=90^{\circ} \mathrm{C}$

\begin{tabular}{|c|c|c|c|c|}
\hline $\mathbf{h}, \mathbf{m m}$ & $\mathbf{Q}, \mathbf{k W}$ & $\mathrm{K}, \mathrm{W} /(\mathbf{M} 2 \cdot \mathrm{K})$ & $\eta э \phi$ & $\mathrm{F}, \mathrm{m} 2 / \mathrm{m}$ \\
\hline 2,00 & 70,46 & 886,4 & 0,412 & 0,372 \\
\hline 2,25 & 71,37 & 828,0 & 0,416 & 0,405 \\
\hline
\end{tabular}




\begin{tabular}{|l|l|l|l|l|}
\hline 2,50 & 72,10 & 777,8 & 0,419 & 0,438 \\
\hline 2,75 & 72,70 & 734,9 & 0,422 & 0,469 \\
\hline 3,00 & 74,30 & 695,6 & 0,424 & 0,500 \\
\hline
\end{tabular}

In Figures 4 and 5, there are graphs of the dependence of the heat output and the heat transfer coefficient on changes in the height of the fins.

$$
\mathrm{Q}, \mathrm{kW}
$$

80

70

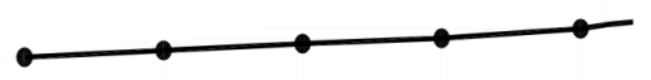

60

50

40

30

20

10
2.0
2.25
2.5
2.75
3.0
$\mathrm{H}, \mathrm{mm}$

Fig. 3. 4. The heat output of the heat exchanger as a function of the height of the ribs. I - with $i_{1}=$ $55^{\circ} \mathrm{C}$, II - with $\boldsymbol{t}_{1}^{\prime}=90^{\circ} \mathrm{C}$.

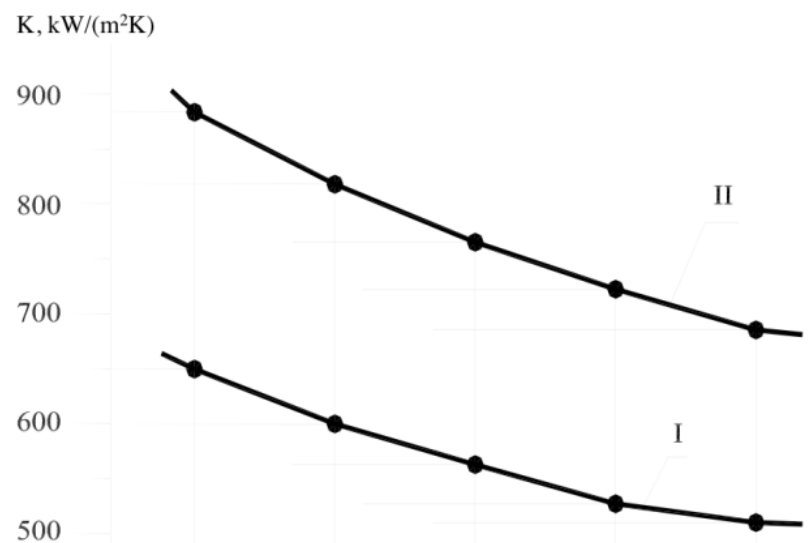
2.0
2.25
2.5
2.75
3.0
$\mathrm{H}$, mm

Fig. 3. 5. The change in the heat transfer coefficient from the change in the height of the ribs. $\mathrm{I}-$ with $t_{1}^{\prime}=55^{\circ} \mathrm{C}$, II - with $\boldsymbol{t}_{1}^{\prime}=90^{\circ} \mathrm{C}$. 


\section{Results}

From the generalization of the presented experimental results, the effect of the geometric parameters of fins on the thermal $\mathrm{HE}$ characteristics follows that the studies and calculations performed for HE "water - oil" allow choosing the best allowed one by the manufacturing technology, the finning parameters.

From Figures 1 and 4, it is evident that the finest finning parameters are the smallest possible fin pitch and the maximum possible fin height. In this case, the HE greatest thermal power and the greatest coefficient of thermal efficiency are achieved at the same flow rates of the heat carriers and their temperature at the inlet to the HE.

The efficiency of heat exchange on the surface of the ribs depends on the height of the rib and the pitch between them. With increasing altitude $h$, the value of the heat transfer coefficient $\mathrm{K}$ on the surface of the ribs decreases, and the thermal power $\mathrm{Q}$ increases (Figures 4, 5).

The reduction in $\mathrm{K}$ is less than the increase in the heat exchange surface $\mathrm{F}$, leading to an overall increase in the thermal power $\mathrm{Q}$. The optimum fin height is $(0.2 \ldots 0.5) \mathrm{d}$, the recommended height is $\mathrm{h}=1.5 \ldots 3 \mathrm{~mm}$. A smaller value is proposed for pipes with $\mathrm{d}>10 \mathrm{~mm}$.

Reduction of the fin step leads to a decrease in convective heat transfer, since at the base of the ribs zones with weak circulation of the flow are formed, this leads to an increase in the thermal power (Figure 1 and 2). The optimum step of the fins $S=(0.05 \ldots 0.3)$ d, the recommended value by the results of tests is $\mathrm{S}=1.5 \ldots 2.5 \mathrm{~mm}$.

The material and the thickness of the fin significantly influence the intensity of heat transfer. The higher the thermal conductivity of the material, the greater the heat transfer coefficient. Therefore, the use of pipes with finning by the DC method from copper and brass is justified from the point of view of heat exchange and improvement of the mass characteristics of HE. With an edge height $\mathrm{h}=3 \mathrm{~mm}$ and $\mathrm{S}=1.5 \mathrm{~mm}$, its optimum thickness is $0.75 \mathrm{~mm}$, which ultimately leads to an improvement in the thermal characteristics of HE.

The increase in thermal efficiency is due to:

- Using the DC method that allows to observe an increase in the heat exchange surface up to 14 times;

- Use of the optimal geometry of the heat exchange surface;

- The absence of a narrowing of the pipe cross-section, which is characteristic for finning by the rolling method;

- The possibility of obtaining a minimum fin pitch, as well as the minimum residual wall thickness of the finned tube (up to $0.4 \mathrm{~mm}$ ), which is determined by its operational strength.

The obtained results of experimental studies are important for assessing the efficiency and reliability of the applied designs of coil heat exchangers in the fields of domestic and foreign engineering industry.

\section{Discussion}

The The results of studies on increasing heat transfer efficiency reveal that the HE coiled tubing from finned tubes has a thermal efficiency higher by $28-40 \%$ than similar HEs made from smooth pipes, while they meet other requirements, such as overall dimensions, compactness, reliability and reduction of harmful toxic emissions into the atmosphere during their manufacture. In the course of experimental studies, it is established that the finning formed by the DC method on the pipes allows them to bend with a bending radius smaller than for a smooth tube, since often located fins perform the function of stiffening 
elements (Fig. 5.1). This feature allows us to wind small-sized coils of any size, and on their basis to develop small-sized effective HE designs. The production of coils by simultaneous winding of two branches of a bent finned tube (Fig.5.2b) allows expanding the area of using coils in multi-pass, the HE modular with one-way inlet and outlet of the cooling medium, reducing the dimensions, HE mass, and simplifying their installation in hydraulic systems of power and process machines.

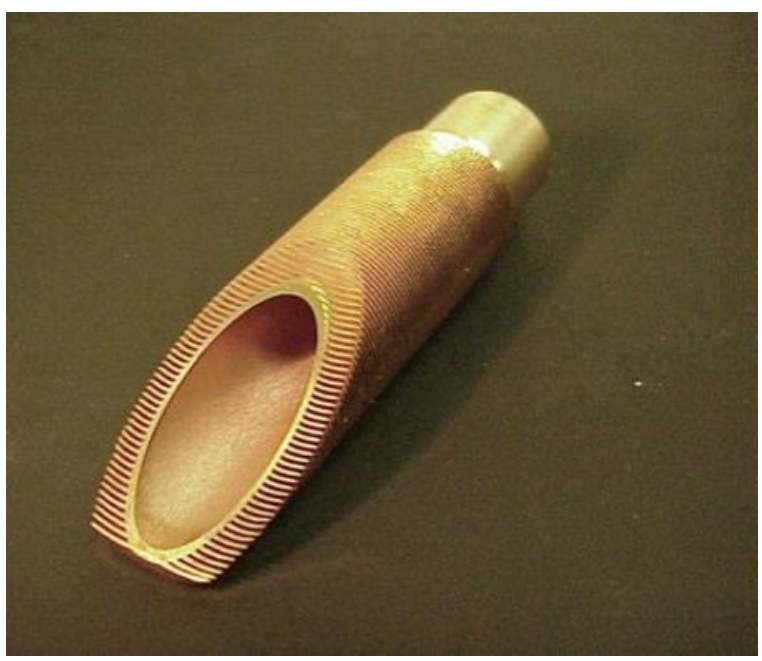

Fig. 5.1. A cut of finned heat exchange pipe obtained by the DC method.

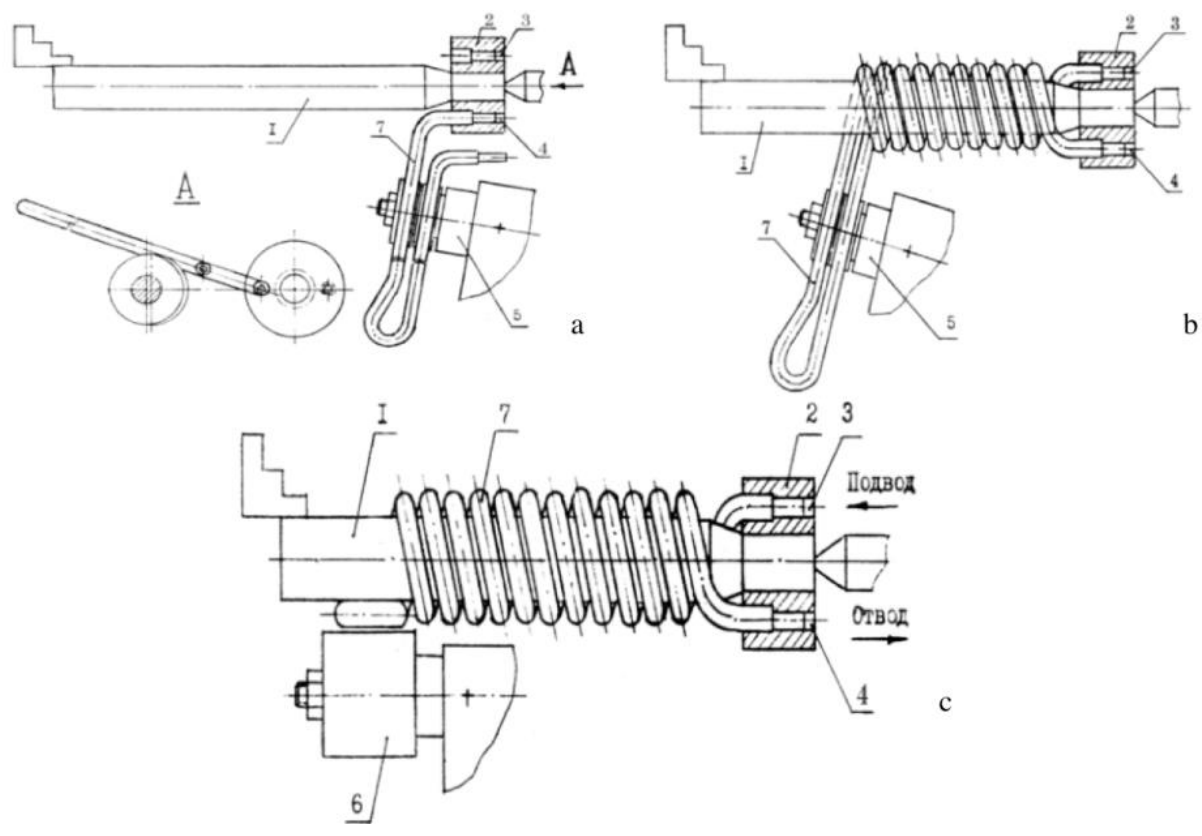

Fig. 5. 2 The method for manufacturing coils from finned tubes: a - fixing the ends of the finned tube in the holes of the head; $b$ - simultaneous winding of two branches of the pipe; $v$ - laying the loop after winding the coil; 1 - the mandrel; 2 - the head; 3 and 4 - holes; 5 - a directing roller; 6 - the smooth roller; 7 - the finned tube. 
These features allowed us to develop designs and technologies for the production of efficient and reliable TAs used in production lines for the production of power and process machines (Figures $6.1-6.8$ ).

The minimum number of soldered pipe joints is provided in the HE coil constructions (Figure 2) (for example, two solder joints instead of 48 and 64 depending on the size for tubular HE), which reduces harmful toxic emissions into the atmosphere during their manufacture.

Industrial introduction of coil HE being developed on the basis of research results allowed to significantly increase the reliability and efficiency of machines for the production of VAZ vehicles and completely refuse to purchase such $\mathrm{HE}$ abroad, and also to protect the environment from harmful industrial emissions.

\section{Conclusion}

Based on the results of development and research, modular coil heat exchangers have been constructed (Figures $6.1-6.8$ ). The designs of heat exchangers and manufacturing technology are protected by RF patents (pp. 19-21 of the literature used). Development and research are aimed at refusing to purchase HE for imports.

Development and research provides an economic benefit from the replacement of one HE unit of the OCW type (VICKERS, Germany) by domestic ones, which is of 100-250 thousand rubles, depending on the type (in prices as of December 1, 2017).

The results of development and research are detailed by the author in the monograph "Physical and mathematical modeling of heat transfer processes in mechanical systems". Designs of the coiled HE from finned tubes, developed on the basis of research results, are presented in Figures $6.1-6.8$.

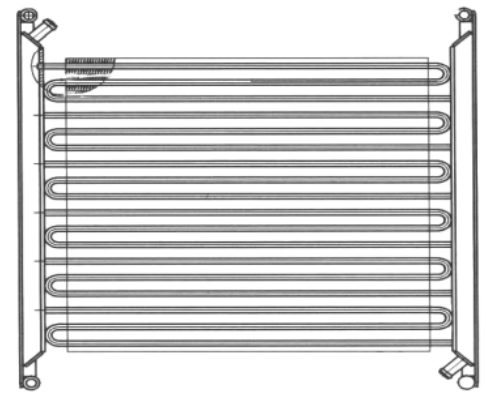

Fig. 6.1 Radiator cooling system ICE

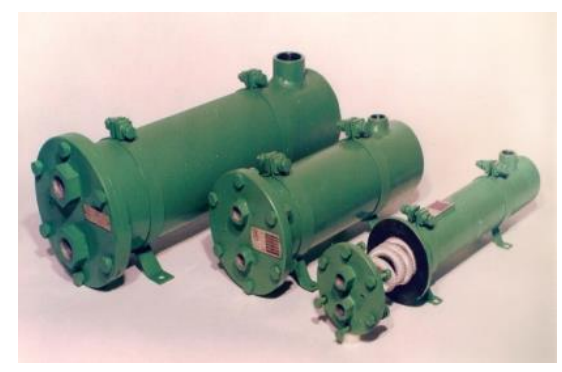

Fig. 6.3 Heat exchangers with coiled radiators from finned tubes for mechanical systems of industrial equipment (types: $\mathrm{a}, \mathrm{b}, \mathrm{c})$; $\mathrm{a}-\mathrm{Du} 15$; b-Du 20; c-Du 40

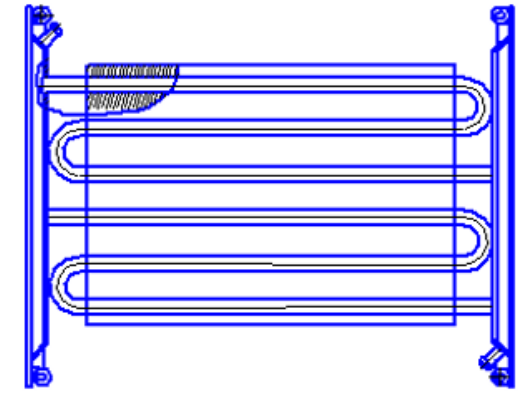

Fig. 6.2 Radiator of lubrication system ICE

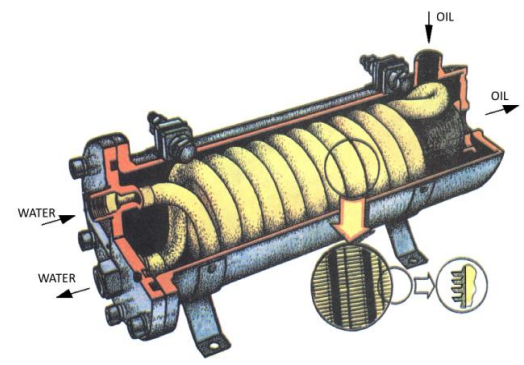

Fig. 6.4 Heat exchanger with single coil from finned tube (type b). 


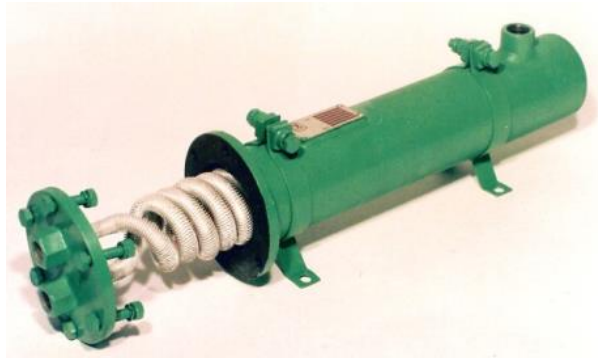

Fig. 6.5 Heat exchanger with single coil from finned tube (type a).

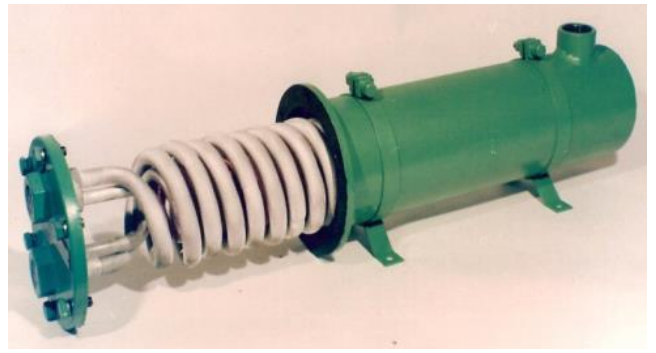

Fig. 6.6 Heat exchanger with double coil from finned tubes (type c).

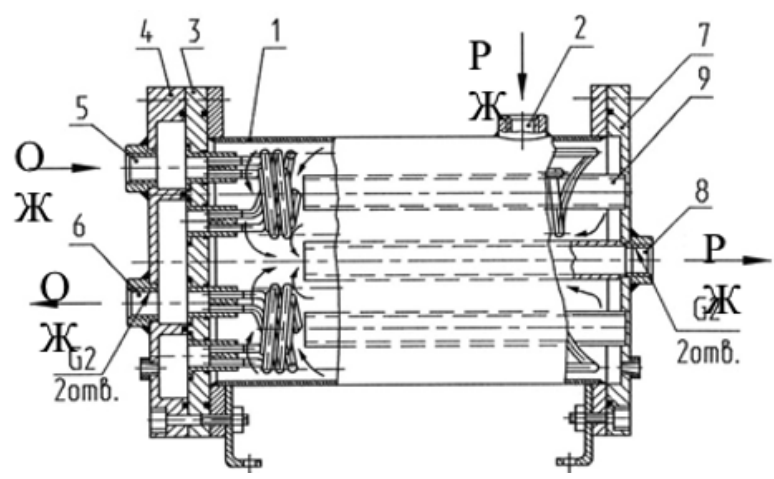

Fig. 6.7 Heat exchanger with a coil radiator from finned tubes - Du 50 (4 Modular): 1 - housing; 2 and 8 - openings for the supply and removal of the Cooling Fluid; 4 - a forward flange; 5 and 6 - the holes for the supply and removal of the Cooling Fluid; 7 - a back flange; 9 - guides; 3 - a flange housing.

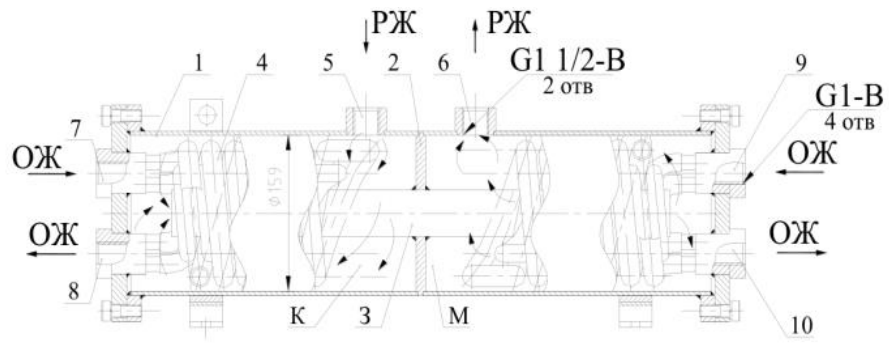

Single-sided coolant supply and outlet scheme

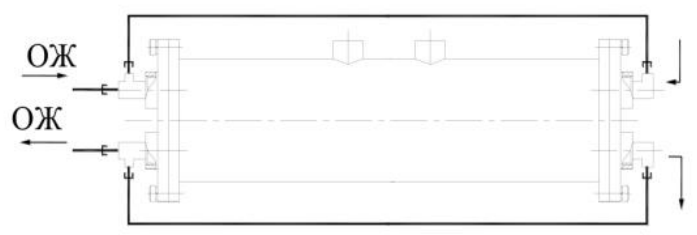

Fig. 6.8 Heat exchanger with a coil radiator from finned tubes - Du 40 (2 Modular): 1 - housing; 2- a partition; 3 - a pipe; 4 - a radiator; 5 - a hole for the supply of working fluid into the cavity K; $6-\mathrm{a}$ hole for the removal of working fluid from the cavity $\mathbf{M} ; 7$ and 8 - holes for supply and removal of coolant; 9 and 10 - openings for supply and removal of a coolant; $\mathrm{K}$ and $\mathrm{M}$ - cavities. 
Table 4. Technical characteristics of heat exchangers.

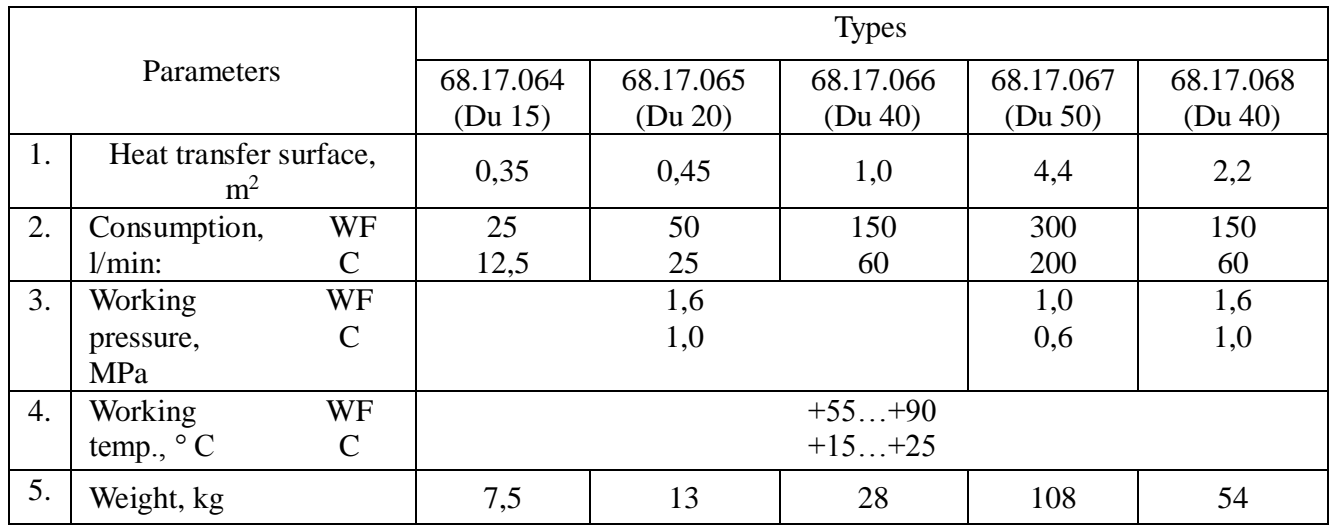

\section{References}

1. N. N. Zubkov Technology of Mechanical Engineering, 1, 19-26 (2001)

2. P. F. Zibrov, A.V. Vasiliev, N. S. Chernov, Physical and mathematical modeling of heat exchange processes in mechanical systems (TSU, Togliatti, 2013)

3. D. Kern, A. Kraus, Development of the heat transfer surface (Energia, Moscow, 1977)

4. E.K. Kalinin, G.A. Dreitser, S.Ya. Yarkho, Intensification of heat transfer in channels (Mechanical Engineering, Moscow, 1992)

5. L. M. Kovalenko, A. F. Glushkov, Heat exchangers with intensification of heat transfer (Energoatomizdat, Moscow, 1986)

6. S.S. Kutateladze, Fundamentals of the theory of heat transfer (Nauka - Siberian Branch, Novosibirsk, 1970)

7. V. K. Migay, Increasing the efficiency of modern heat exchangers (Energia, Leningrad, 1980)

8. E. M. Chizhevskaya, Yu. M. Brodov, R. Z. Saveliev, Proceedings of Universities Energy, 5, 109-112 (1984)

9. B. L. Trotsky, E. A. Sudareva, Fundamentals of design of ship power plants (Sudostroeniye, Leningrad, 1987)

10. M. Ya. Belenky, M. Ya. Gotovsky, B. M. Lekah, TVT, 29(6), 1142-1147 (1991)

11. V. F. Yudin, Heat transfer of cross-ribbed pipes (Mechanical Engineering, Leningrad, 1982)

12. V. F. Yudin, L. S. Tokhtarova, Power Engineering, 1, 19-21 (1974)

13. L. A. Benan, S. Yu. Michael, A.S. 193897 USSR, M.C.3. In the 21D 11/06. Method of manufacturing coils. No. 1045140 /25-27, Bulletin 29 (1981)

14. E. P. Cherpillo, B. G. Buneev, A.S. 548167 USSR, M.C.3.3 B21C 37/22. Method of tube finning. №2102919 / 22-02, Bulletin 23 (1985)

15. V. I. Gurov, M. V. Lepork, A. A. Suchkov, A.S. 597466 USSR, M.Kl.2 B21D 11/06. Method of manufacturing coils. No. 2412675 / 25-27, Bulletin 6 (1975)

16. F. P. Kirpichnikov, E. M. Panfilov, A.S. 923661 USSR, M.C. 3 D21C 37/20, B21H 7/18. Method of manufacturing ribbed pipes. No. 2988861 /25-27, Bulletin 16 (1980) 
17. V. I. Gurov, Yu. M. Dorfman, A.S. 940920 USSR, M.C. 3 B21D 11/06. Device for the manufacture of coils. No 2820642/25-27, Bulletin 25 (1982)

18. A. I. Sardak, P. A. Barabash, A.S. 1269874 USSR, M.S. 3 B21C 37/26, F28F 1/36. Method for manufacturing finned heat-exchanging tubes. No. 3947981/25-27, Bulletin 42 (1986)

19. N. S. Chernov, V. A. Kachalov, A. I. Ovchinnikov, Patent 2087236 of the Russian Federation, IPC 7 B 21D 53/06, 11/06. The method of manufacturing coils from finned tubes, Bulletin 23 (2000)

20. N. S. Chernov, V. P. Muranovsky, Patent 2176061 of the Russian Federation, IPC 7 F 28 D 7/02. Heat exchanger. №2000109329/06, Bulletin 32 (2001)

21. N. S. Chernov, Patent 2263265 of the Russian Federation, IPC 7 F 28 D 7/02. Heat exchanger. №2004105333/06, Bulletin 30 (2005)

22. V. Gnielinski, E. S. Gaddis, Umlenkblechen (Verfahrentechnik), 12(4), 211-217 (1978)

23. G. Hailer, Chemie Ingenieur Technik, 10 (1974)

24. C. I. King, I. R. Davis, Mach. Des., 42(22), 42-43 (1970)

25. R. G. Malek, Hydrocarbon Processing, 128-130 (1973)

26. H. Schnaike, A. Cooper, Hansa-Schiffahrt-Schiffbau-Haffen, 114(9), 842-845 (1977)

27. N. N. Zubkov Russian Engineering Research, 10, 13-20 (1994) 